\title{
Problema de asignación para operaciones de comando dual en un almacén con un layout transversal y de espina de pescado
}

\author{
Assignment Problem for Dual-Command Operations in a Warehouse with a Transversal and \\ Fishbone Layout \\ Isidro J. González-Hernández ${ }^{a}$, Rafael Granillo-Macías ${ }^{b}$, Isaías Simón-Marmolejo ${ }^{b}$, Diana \\ Sánchez-Partida ${ }^{c}$, Francisca Santana-Robles ${ }^{b}$, Emmanuel Juárez-García ${ }^{c}$
}

\begin{abstract}
:
The processes related to the products storage have become a strategic element of the supply chain management, that the efficiency of the whole chain has been related to the performance of the warehouse operations and particularly, by the operations of storage and retrieval of products. In this paper, we address an assignment problem for dual command operations (storage-collection) for a warehouse with a transversal and fishbone layout design. A linear programming model was used to minimize the distances traveled in all the dual cycles. The contribution of this work is to the state of the art that allows helping the supply chain managers to make decisions to choose the best layout design in a warehouse where it carries out dual command operations.
\end{abstract}

\section{Keywords:}

Warehousing, dual command, assignment problem, warehouse layout

\section{Resumen:}

Los procesos relacionados con el almacenamiento de productos se han convertido en un elemento estratégico de la administración de la cadena de suministro, ya que la eficiencia de toda la cadena se ve afectada por el desempeño de las operaciones del almacén y particularmente, por las operaciones de almacenamiento y recuperación de bienes. En este trabajo, se aborda un problema de asignación para operaciones de comando dual (almacenamiento-recolección) para un almacén con un diseño de layout en forma transversal y fishbone. Se utilizó un modelo de programación lineal para minimizar las distancias recorridas en los ciclos duales. La aportación de este trabajo es al estado del arte que permita ayudar a los administradores de la cadena de suministro a la toma de decisiones para elegir el mejor diseño del layout en un almacén donde se llevan a cabo operaciones de comando dual.

\section{Palabras Clave:}

Almacenamiento, comando dual, problema de asignación, layout del almacén

\section{Introducción}

Hoy en día, las empresas se están enfrentando a un mercado más competitivo y tecnológico (e-comercio e Internet de las cosas), debido a esta competencia, la administración de la cadena de suministro desempeña uno de los factores más importantes tanto en la satisfacción del cliente como la rentabilidad de los negocios. 1 De acuerdo con los principios de la administración de la cadena de suministro, las empresas tienen como objetivo cumplir con los pedidos de los clientes, además de optimizar aspectos como el tiempo de entrega, minimizar costos operativos y de distribución, incrementar la flexibilidad y la confiabilidad, y minimizar

\footnotetext{
a Autor de Correspondencia, Universidad Autónoma del Estado de Hidalgo, Escuela Superior de Cd. Sahagún - Ingeniería Industrial, Tepeapulco, Hidalgo, México. https://orcid.org/0000-0003-2805-6674, Email: igonzalez@uaeh.edu.mx

${ }^{\mathrm{b}}$ Universidad Autónoma del Estado de Hidalgo, Escuela Superior de Cd. Sahagún - Ingeniería Industrial, Tepeapulco, Hidalgo, México. (Granillo) https://orcid.org/0000-0002-1015-667X. (Simón) https://orcid.org/0000-0003-2116-6192. (Santana) https://orcid.org/0000-0002$3301-9790$

c Universidad Popular Autónoma del Estado de Puebla, Logística y Dirección de la Cadena de Suministro, Puebla, Puebla, México. 
en el consumo de energía. El logro de todos estos objetivos tiene una relación directa con la toma de decisiones estratégicas enfocadas a determinar el diseño y número de almacenes o centros de distribución (CEDIS), así como las decisiones tácticas y operativas enfocadas a la administración de las operaciones de estas instalaciones, de tal forma que, una disposición (layout) y una administración eficiente de un almacén o CEDIS es una estrategia clave para lograr la competitividad de las empresas. 2,3

Un almacén o CEDIS es una instalación intermedia entre proveedores y clientes que tiene el papel importante de mantener las operaciones diarias de la cadena de suministro para satisfacer las demandas del cliente (nivel de servicio al cliente). Por lo tanto, las principales operaciones que se desarrollan en un almacén o CEDIS son recepción, clasificación, almacenamiento, preparación de pedidos y el envío de pedidos, entre estas operaciones, el almacenamiento de productos y la preparación de pedidos se encuentran entre las actividades que consumen más recursos. De tal forma que, la administración de un almacén o CEDIS es el arte de almacenar y mover el inventario a través de estas instalaciones para que el flujo de productos se optimice. 3-5

Las operaciones de un almacén o CEDIS es uno de los principales problemas a los que se enfrentan los administradores de la cadena de suministro. Primero, un diseño efectivo del almacén o CEDIS puede reducir claramente los costos de operación y logísticos. Segundo, un buen método para asignar la ubicación de almacenamiento de los productos puede reducir los tiempos de almacenamiento y las actividades de preparación de pedidos, así como, disminuir las distancias totales de viaje (estiva y recolección) y disminuir la congestión en el flujo de productos. Tercero, una política de inventarios eficiente puede reducir los niveles de inventario y por consecuencia se reducen los costos de mantener inventario. 6

Cardona et al. mencionan que los almacenes de todo el mundo tienen el mismo diseño, conocido como diseño tradicional, que se caracteriza por la disposición de pasillos paralelos, ortogonales a las paredes como se muestra en la Figura 1. 7

Además de los diseños tradicionales en el 2009, Gue y Meller propusieron un diseño no tradicional, llamado diseño de espina de pescado (fishbone layout, por su nombre en inglés) que llamó la atención de administradores y académicos de la cadena de suministro, como se muestra en la Figura 2. 8
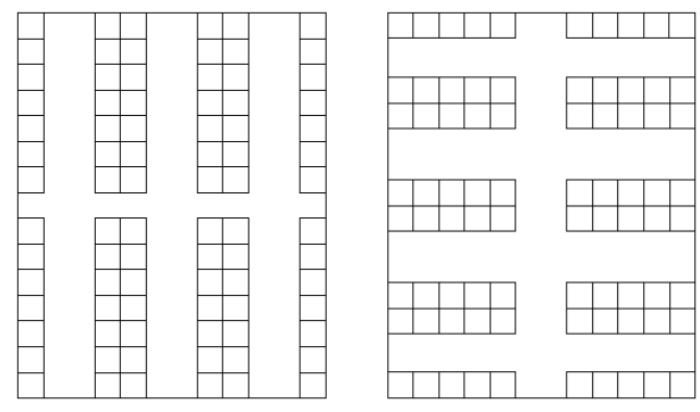

Figura 1. Layout longitudinal o transversal en la zona de almacenamiento (tomada de Ghiani et al. (2013)) 9

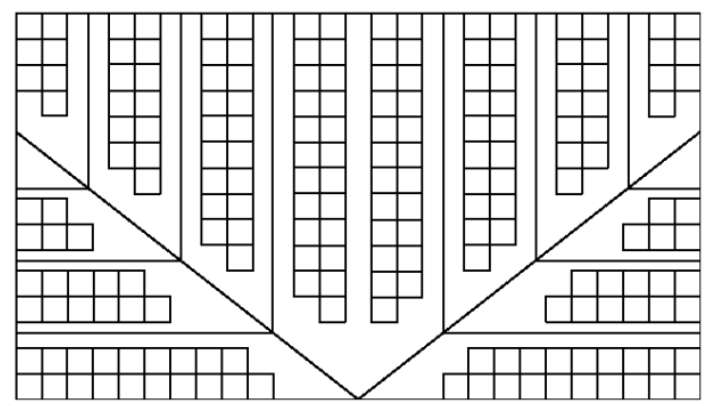

Figura 2. Layout Fishbone en la zona de almacenamiento (tomada de Ghiani et al. (2013)) 9

Por lo tanto, las decisiones con respecto a la distribución - layout de un almacén o CEDIS son una parte integral del proceso de diseño del almacén. Dukic and Opetuk 10 mencionan que hay dos tipos de problemas en la toma de decisiones para el layout de un almacén o CEDIS. El primer problema tiene que ver con la decisión de dónde ubicar las distintas áreas (recepción, clasificación, almacenamiento, recolección, envío, etc.), a este problema se suele llamar problema de distribución de planta (facility layout problem, por su nombre en inglés), el objetivo común es minimizar el costo de manejo, es decir, minimizar las distancias de viaje. El segundo problema, se refiere a la ubicación del equipo, el espacio de almacenamiento, las rutas para almacenamiento y recolección, etc., dentro de los departamentos. Por lo general, se denomina problema de configuración de pasillos (internal layout design problem, por su nombre en inglés), el objetivo de este problema es encontrar un layout óptimo (o al menos uno bueno) para el área de almacenamiento o preparación de pedidos, en la mayoría de los casos, el criterio es la distancia de viaje o tiempo de viaje.

Por otra parte, las operaciones de almacenamiento y recolección se pueden realizar desde dos enfoques: (1) operaciones de ciclo de comando único y (2) operaciones de ciclo de comando dual. En el ciclo de comando único, un operario realiza sólo el 
almacenamiento o la recolección de productos en cada viaje al almacén, por lo tanto, un operario (montacargas) que opera bajo un protocolo de ciclo de comando único se detiene al menos la mitad del tiempo, ya que debe volver constantemente vacío al área de recibo para cargar uno o varios pallets o bien viajar vacío para recuperar uno o varios pallets para envío, como se muestra en la Figura 3. En un ciclo de comando dual, un trabajador debe realizar dos operaciones diferentes: primero visita una ubicación para un almacenamiento y luego trasladarse a otra ubicación para una recolección, como se muestra en la Figura 4. Los comandos duales son más eficientes con respecto a los viajes, pero requieren operaciones de recepción (almacenamiento) y envío (recolección) simultáneas y sistemas de Tecnologías de la Infamación avanzados para dirigir a los trabajadores a las ubicaciones seleccionadas. 8,11

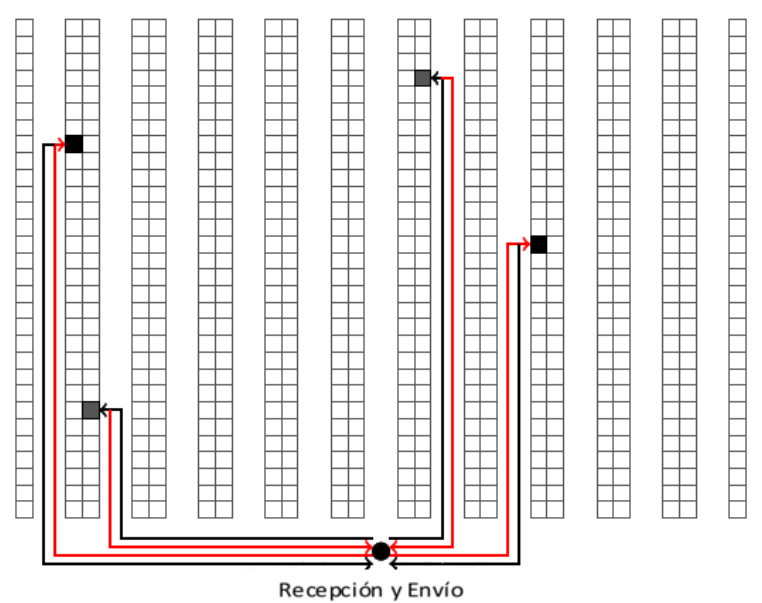

Figura 3. Ciclo de comando único. Tiempo muerto en línea roja (tomada de Bartholdi and Hackman (2017) 11

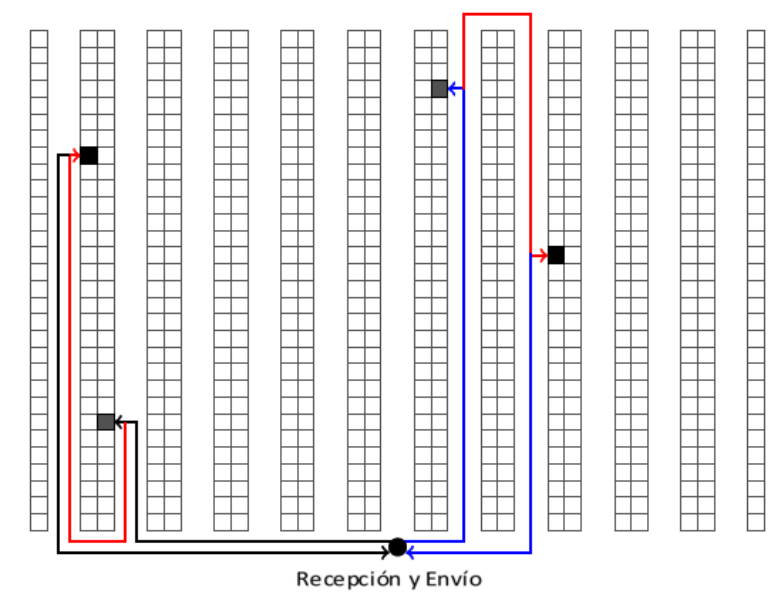

Figura 4. Ciclo de comando dual. Tiempo muerto en línea roja (tomada de Bartholdi and Hackman (2017) 11
Bajo este contexto, el presente trabajo aborda un problema de asignación para las operaciones de almacenamiento y recolección (operaciones de ciclo dual o comando dual) en un almacén de producto terminado, en donde se considera en un primer caso una configuración de layout transversal con puertas de entrada y salida en lados opuestos, en el segundo caso se considera una configuración de layout en forma de "Fishbone". Para resolver la instancia se utilizará un modelo de programación lineal. La principal contribución de este trabajo es al estado del arte que permita facilitar la toma de decisiones a los administradores de la cadena de suministro para elegir la mejor configuración del layout en un almacén donde se lleven a cabo tareas de ciclo dual (almacenamiento y recolección).

\section{Revisión de la literatura}

Yener y Yazgan examinan tres problemas populares de un almacén: diseño del almacén, asignación de almacenamiento y preparación de pedidos (order picking, por su nombre en inglés). 12

De tal forma que, en este trabajo se investiga la efectividad del diseño de un almacén para determinar el tiempo promedio de recolección de pedidos y la distancia recorrida utilizando una técnica de minería de datos. En primer lugar, se investiga la efectividad de las diferentes alternativas de diseño de almacenes que existen. En segundo lugar, proponen un modelo de asignación cuadrática de enteros mixtos y el uso de un algoritmo de escalamiento multidimensional para resolver un problema de asignación de almacenamiento. Finalmente, examinan la efectividad del rendimiento del sistema con el uso de un modelo de simulación desarrollado para diferentes opciones de diseño de almacenes.

Zhang et al. abordan un problema de coordinación entre la planeación de la producción y la administración de almacenes. 6

Para resolver el problema presentan una estrategia integrada que combina el diseño del almacén con el problema del tamaño de lote capacitado, es decir, combinan un problema de asignación de ubicación de almacenamiento dedicado con el problema de tamaño de lote capacitado en un solo modelo matemático que minimiza el costo total del viaje, el espacio de almacenamiento reservado, el manejo, la producción, el inventario y los costos de configuración. Desarrollan un modelo de programación lineal entero mixto para formular el problema de optimización. Para resolver el problema a gran escala proponen un nuevo enfoque heurístico lagrangiano de relajación y reparación (Lagrangian relax-and-fix heuristic, por su nombre en inglés). 
Cardona et al. proponen un método para generar un diseño tridimensional detallado para un layout de espina de pescado o fishbone. El método considera las dimensiones de las aberturas, el ancho del pasillo, las velocidades de los montacargas, el costo de arrendamiento, el costo del mantenimiento del área de almacén y el costo de manejo de materiales con el objetivo de satisfacer una capacidad de almacenamiento deseada. Además, proporcionan un procedimiento de optimización basado en un algoritmo genético para resolver el modelo de optimización. 7

Horta et al. proponen un enfoque para el diseño del layout de un almacén que alimenta la operación de distribución justo a tiempo en un ambiente de cruce de mulle (cross docking, por su nombre en inglés), el cual requiere la asignación de espacios en piso para atender las demandas de las tiendas o clientes. El flujo de productos comienza en los muelles de recepción, después se desplaza al espacio asignado de la tienda que requiere el producto y visita tantas ubicaciones en el piso en función de las tiendas que requieren ese producto hasta que el contenedor esté vacío. De tal forma que, desarrollan un modelo de programación entera para asignar las ubicaciones a las tiendas, minimizando la distancia total recorrida dentro del almacén. 13

Arnaout et al. abordan un problema para el diseño de un almacén con niveles múltiples, que implica la asignación de los artículos a celdas y niveles con el objetivo de minimizar los costos de transporte. Los autores adaptan un algoritmo de optimización de colonias de hormigas para resolver el problema, además evalúan su desempeño del algoritmo comparando sus soluciones con las soluciones obtenidas utilizando algoritmos genéticos. 14

De acuerdo a Yener y Yazgan, en su revisión de la literatura mencionan que los enfoques que se han empleado para resolver el diseño del layout de un almacén o CEDIS han sido los siguientes: Modelo matemático, Sistema de soporte a las decisiones (Decision Support System, por su nombre en inglés), Algoritmos heurísticos, Minería de datos, Escalamiento multidimensional (Multidimensional Scaling, por su nombre en inglés) y Técnicas de simulación. 12

Por otra parte, Ballestín et al. abordan un problema de almacenamiento y recuperación en un almacén con almacenamiento aleatorio $y$ las operaciones de almacenamiento se realizan por medio de un conjunto de montacargas con capacidades diferentes (lo que significa que no todos los montacargas pueden acceder al mismo conjunto de ubicaciones). 4

El problema consiste en asignar ubicaciones a las operaciones, asignar montacargas a las operaciones y programar las operaciones asignadas a un montacargas para minimizar el tiempo empleado, de tal forma que, el problema se resuelve heurísticamente descomponiéndolo en tres sub-problemas.

Hachemi et al. abordan un problema de secuenciación para operaciones de ciclo dual, es decir, almacenamiento y recuperación, donde un producto requerido puede estar en varias ubicaciones de rack y hay un conjunto de ubicaciones vacías. De tal forma que, las ubicaciones de recuperación y almacenamiento no se conocen a priori. El problema de secuencia consiste en determinar para cada ciclo dual las ubicaciones de almacenamiento y recuperación que satisfacen las solicitudes de recuperación y almacenamiento. El objetivo es encontrar una ubicación vacía de todas las ubicaciones vacías y una ubicación de recuperación de todas las ubicaciones que contengan los elementos solicitados de tal manera que den el tiempo mínimo de viaje. El problema de optimización está formulado en términos de programación entera y aplican una heurística. 15

Además, otros autores han abordado la programación de maquinas (o grúas) para sistemas de almacenamiento/recuperación automatizados (Automated Storage and Retrieval Systems (AS/RS), por su nombre en inglés) con el objetivo de minimizar los tiempos de viaje entre las operaciones de almacenamiento y recolección. Por ejemplo, Ghomri y Sari abordaron este problema utilizando modelación analítica, Yang et al. desarrollaron un algoritmo de búsqueda en vecindario variable (variable neighborhood search, por su nombre en inglés) para resolver los problemas de gran tamaño, Brezovnik et al. utilizaron un algoritmo de colonia de hormigas. 16-18

\section{Descripción del problema}

La velocidad a la que se puede realizar la operación de almacenamiento y recolección de productos depende en gran medida de los lugares donde se encuentran o deben situarse los bienes para almacenar o recuperar. De tal forma que, la aplicación de políticas de control debe administrar el sistema en la medida en que logre minimizar las distancias de viaje. Por lo tanto, para asignar pares de viajes almacenamiento-recolección, se puede abordar desde un enfoque de un problema de asignación.

Para la formulación de este problema se supone que el problema de asignación del ciclo dual no tiene una solución permanente debido al constante cambio de la demanda. En cada turno de trabajo se pueden recibir diferente número de órdenes que deben ser enviadas lo antes posible al cliente. En este problema se consideran 3 turnos al día y cada turno debe de hacer pares de operaciones, es decir, un almacenamiento y una recolección. El número de órdenes, tanto de 
almacenamientos como recolecciones a atender ya están registradas desde un día antes. Por lo tanto, el problema se convierte en un problema Multiperiodo. Para este problema, el turno uno debe realizar 11 ciclos dual, el turno dos tiene que trabajar 9 ciclos dual y el turno tres tiene programado 5 ciclos dual. Además, el problema considera un diseño de layout del almacén en forma transversal, como se muestra en la Figura 5, así como un layout en forma de fishbone, como se muestra en la Figura 6. En ambas figuras (5 y 6), S1, S2, ..., S25 son las ubicaciones donde se debe almacenar el producto. R1, $\mathrm{R} 2, \ldots, \mathrm{R} 25$ son las ubicaciones donde se debe recolectar el producto, además cada cuadro de las figuras representa una unidad de distancia en el almacén. El objetivo del problema es identificar que diseño de layout proporciona la menor distancia recorrida en los tres turnos.

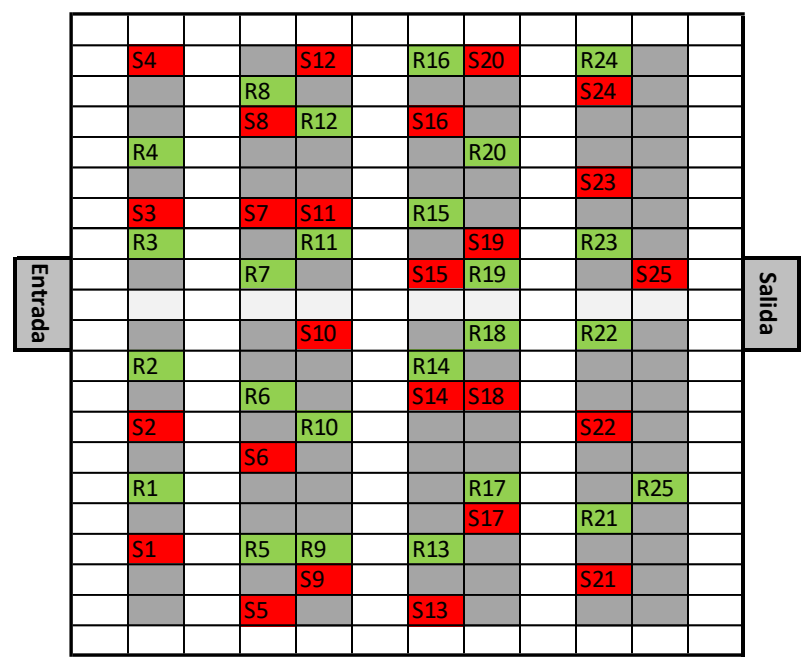

Figura 5. Diseño del layout del almacén en forma transversal

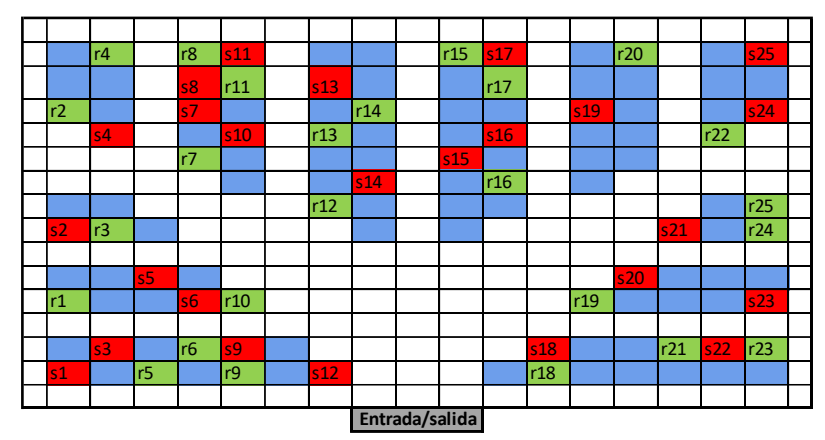

Figura 6. Diseño del layout del almacén en forma fishbone

\section{Formulación del problema}

Se plantea un modelo de programación lineal para minimizar la distancia total recorrida.

Índices:

$$
\begin{array}{ll}
i=1,2,3, \ldots, m & \begin{array}{l}
\text { Número de tareas para } \\
\text { almacenamiento. }
\end{array} \\
j=1,2,3, \ldots, n & \text { Número de tareas para recolección. } \\
k=1,2,3 & \text { Número de turnos de trabajo al día. }
\end{array}
$$

Parámetros:

$T_{k} \quad$ Cantidad de ciclos duales (almacenamientorecolección) en el turno $k$.

$d_{i j} \quad$ Distancia recorrida entre la ubicación de almacenamiento $i$ y la ubicación de recolección $j$.

Variables de decisión:

$x_{i j} \quad 1$ si el operario (o montacargas) realiza el almacenamiento $i$ y después la recolección $j$ en el turno 1.0 de lo contrario.

$y_{i j}$

1 si el operario (o montacargas) realiza el almacenamiento $i$ y después la recolección $j$ en el turno 2. 0 de lo contrario.

$z_{i j} \quad 1$ si el operario (o montacargas) realiza el almacenamiento $i$ y después la recolección $j$ en el turno 3. 0 de lo contrario.

Modelo

$$
\min \sum_{i, j} d_{i j} x_{i j}+\sum_{i, j} d_{i j} y_{i j}+\sum_{i, j} d_{i j} z_{i j}
$$

Sujeto a

$$
\begin{aligned}
& \sum_{i} x_{i j}=1 \\
& \sum_{j} x_{i j}=1 \\
& \sum_{i, j} x_{i j}=T_{1}
\end{aligned}
$$




$$
\begin{gathered}
\sum_{i} y_{i j}=1 \\
\sum_{j} y_{i j}=1 \\
\sum_{i, j} y_{i j}=T_{2} \\
\sum_{i} z_{i j}=1 \\
\sum_{j} z_{i j}=1 \\
\sum_{i, j} z_{i j}=T_{3} \\
x_{i j}, y_{i j}, z_{i j} \in\{0,1\}
\end{gathered}
$$

La función objetivo (1) minimiza la distancia total recorrida en los tres turnos de trabajo, la restricción (2), (5) y (8) requiere que cada almacenamiento esté emparejado con alguna recolección en el turno 1, 2 y 3 respectivamente, y la restricción (3), (6) y (9) indica que cada recolección debe estar emparejada con algún almacenamiento en el turno 1, 2 y 3 respectivamente. Las restricciones (4), (7) y (10) indican que sólo se debe realizar la cantidad de ciclos duales (almacenamientorecolección) programados en el turno $k$. Finalmente la restricción (11) indica que las variables de decisión son de tipo binario.

\section{Resultados}

Para resolver el modelo de programación lineal se utilizó el software Lingo, en el Anexo 1 se muestra la programación del problema en el software mencionado. En la Tabla 1 se presentan los resultados de las distancias recorridas en los diferentes turnos para el layout transversal. En la Tabla 2 se presenta la configuración de los ciclos dual para cada turno, donde "S" es almacenamiento y "R" es recolección, el número que le sigue a cada letra es la ubicación donde se debe almacenar y recolectar el producto.

Tabla 1. Resultados de la función objetivo para el layout transversal.

\begin{tabular}{lcc}
\hline Turno & $\begin{array}{l}\text { No. de Ciclos } \\
\text { Dual }\end{array}$ & $\begin{array}{l}\text { Función } \\
\text { Objetivo }\end{array}$ \\
\hline Matutino (T1) & 11 & 531 \\
\cline { 2 - 3 }
\end{tabular}

Vespertino (T2) 9

Nocturno (T3) 5

Tabla 2. Configuración para las actividades de almacenamiento-recolección en el layout transversal.

\begin{tabular}{ll}
\hline Turno & Distancia Recorrida \\
\hline T1( S2, R6) 21.00 & \\
T1( S3, R7) 21.00 & \\
T1( S7, R3) 21.00 & \\
T1( S10, R14) 22.00 & \\
T1( S11, R15) 22.00 & \\
T1( S14, R10) 22.00 & \\
T1( S15, R11) 21.00 & \\
T1( S18, R18) 22.00 & \\
T1( S19, R23) 19.00 & \\
T1( S22, R17) 23.00 & \\
T1( S23, R20) 23.00 & \\
\hline T2( S2, R6) 21.00 & \\
T2( S3, R7) 21.00 & \\
T2( S7, R3) 21.00 & \\
T2( S10, R14) 22.00 & \\
T2( S11, R15) 22.00 & \\
T2( S14, R10) 22.00 & \\
T2( S15, R11) 21.00 & \\
T2( S18, R18) 22.00 & \\
T2( S19, R23) 19.00 & \\
\hline T3( S2, R6) 21.00 & \\
T3( S3, R3) 20.00 & \\
T3( S15, R11) 21.00 & \\
T3( S18, R22) 22.00 & \\
T3( S19, R23) 19.00 & \\
\hline Distancia Total Recorrida & \\
\hline
\end{tabular}

En la Tabla 3 se presentan los resultados de las distancias recorridas en los diferentes turnos para el layout fishbone. En la Tabla 4 se presenta la configuración de los ciclos dual para cada turno, donde "S" es almacenamiento y "R" es recolección, el número que le sigue a cada letra es la ubicación donde se debe almacenar y recolectar el producto.

Tabla 3. Resultados de la función objetivo para el layout fishbone.

\begin{tabular}{lcc}
\hline Turno & $\begin{array}{l}\text { No. de Ciclos } \\
\text { Dual }\end{array}$ & $\begin{array}{l}\text { Función } \\
\text { Objetivo }\end{array}$ \\
\hline Matutino (T1) & 11 & \\
Vespertino (T2) & 9 & 507 \\
Nocturno (T3) & 5 & \\
\hline
\end{tabular}

Tabla 4. Configuración para las actividades de almacenamiento-recolección en el layout fishbone. 


\begin{tabular}{lc}
\hline Turno & Distancia Recorrida \\
\hline T1( S1, R5) 26.00 & \\
T1( S5, R3) 22.00 & \\
T1 ( S6, R6) 19.00 & \\
T1( S9, R10) 14.00 & \\
T1( S10, R13) 24.00 \\
T1( S12, R9) 18.00 \\
T1( S14, R14) 27.00 \\
T1( S18, R19) 11.00 \\
T1( S19, R17) 26.00 \\
T1( S21, R24) 26.00 \\
T1( S23, R23) 25.00 \\
\hline T2( S1, R5) 26.00 \\
T2( S5, R3) 22.00 \\
T2( S6, R6) 19.00 \\
T2( S9, R10) 14.00 \\
T2( S10, R13) 24.00 \\
T2( S12, R9) 18.00 \\
T2( S18, R19) 11.00 \\
T2( S21, R24) 26.00 \\
T2( S23, R23) 25.00 \\
\hline T3( S5, R3) 22.00 \\
T3( S6, R6) 19.00 \\
T3( S9, R10) 14.00 \\
T3( S12, R9) 18.00 \\
T3( S18, R19) 11.00 \\
\hline Distancia Total Recorrida \\
\hline
\end{tabular}

Como se puede observar el layout fishbone arrojo mejores resultados. Cardona et al. mencionan que, la distancia de viaje esperada en un layout fishbone reducirá la distancia hasta un 20\% menor en comparación con los diseños tradicionales. Sin embargo, Bartholdi y Hackman mencionan que en un layout fishbone se requiere de más espacio en comparación de los almacenes tradicionales para compensar el espacio perdido en los pasillos adicionales. 7

\section{Conclusiones}

En este trabajo se abordó un problema de asignación para operaciones de ciclo dual en un almacén o CEDIS (operación que realiza un almacenamiento y después una recolección de productos) en dos diferentes diseños de layout. Los resultados de este trabajo y la literatura muestran que un layout en forma de fishbone puede minimizar hasta un $20 \%$ el recorrido de las distancias en las operaciones de ciclo dual. Esta estrategia del layout en forma de fishbone puede resultar factible para almacenes donde las operaciones se realizan de forma manual o con ayuda de un montacargas. Sin embargo, actualmente la administración de las operaciones de los almacenes 0 CEDIS es utilizar sistemas de almacenamiento y recuperación automatizados (Automated Storage and Retrieval System (AS/RS), por su nombre en inglés) y la tendencia de la Industria 4.0 está orientando estos sistemas a utilizar sistemas de almacenamiento y recuperación automatizados basados en transelevadores o lanzaderas (Shuttle-Based Storage and Retrieval System (SBS/RS), por su nombre en inglés) que transportan en su cuna el Pallet Shuttle (carro eléctrico) y la carga, siguiendo las órdenes lanzadas por el software de gestión de almacenes. En estos sistemas automatizados, el diseño del layout del almacén o CEDIS es de forma longitudinal, es decir, es una estantería de pallets que combina elementos verticales (bastidores) con elementos horizontales (largueros) con lo que se obtiene una estructura altamente estable capaz de albergar en longitud y en altura cargas de elevado peso. Finalmente el objetivo de los administradores y académicos de almacenes es optimizar las operaciones de almacenamiento, recolección y preparación de pedidos en cualquier tipo de sistema de almacenamiento por lo que constantemente surgen nuevas tecnologías, modelos analíticos y metaheurística que ayudan a optimizar estas operaciones.

\section{Referencias}

[1] González-Hernandez IJ, Martínez-Flores JL, Sánchez-Partida D, et al. Relocation of the distribution center of a motor oil producer reducing its storage capacity: A case study. Simul Trans Soc Model Simul Int. Epub ahead of print 2018. DOI: 10.1177/0037549718825299.

[2] Ekren BY, Akpunar A, Sari Z, et al. A tool for time, variance and energy related performance estimations in a shuttle-based storage and retrieval system. Appl Math Model 2018; 63: 109-127.

[3] Wisittipanich W, Kasemset C. Metaheuristics for Warehouse Storage Location Assignment Problems. In: CMUJ NS Special Issue on Logistics and Supply Chain Systems. 2015, pp. 361-378.

[4] Ballestín F, Pérez Á, Quintanilla S. A multistage heuristic for storage and retrieval problems in a warehouse with random storage. Int Trans Oper Res 2017; 00: 1-30.

[5] Zhang R-Q, Wang M, Pan X. New model of the storage location assignment problem considering demand correlation pattern. Comput Ind Eng 2019; 129: 210-219.

[6] Zhang G, Nishi T, Turner SDO, et al. An integrated strategy for a production planning and warehouse layout problem: Modeling and solution approaches. Omega 2017; 68: 85-94.

[7] Cardona LF, Soto DF, Rivera L, et al. Detailed design of fi shbone warehouse layouts with vertical travel. Int J Prod Econ 2015; 170 : $825-837$.

[8] Gue KR, Meller RD. Aisle configurations for unit-load warehouses Aisle configurations for unit-load warehouses. IIE Trans 2009; 4: 171182 .

[9] Ghiani G, Laporte G, Musmanno R. Introduction to Logistics Systems Management. Second. Wiley, 2013. 
[10] Dukic G, Opetuk T. Warehouse Layouts. In: Manzini R (ed) Warehousing in the Global Supply Chain: Advanced Models, Tools and Applications for Storage Systems. Springer-Verlag London, 2012, pp. 55-69.

[11] Bartholdi JJ, Hackman ST. Warehouse \& Distribution Science Release 0.98. 0.98. Atlanta, GA, USA: The Supply Chain \& Logistics Institute, 2017.

[12] Yener F, Yazgan HR. Optimal warehouse design: Literature review and case study application. Comput Ind Eng 2019; 129: 1-13.

[13] Horta M, Coelho F, Relvas S. Layout design modelling for a real world just-in-time warehouse. Comput Ind Eng 2016; 101: 1-9.

[14] Arnaout JP, ElKhoury C, Karayaz G. Solving the multiple level warehouse layout problem using ant colony optimization. Oper Res 2017; 1-18.

[15] Hachemi K, Sari Z, Ghouali N. A step-by-step dual cycle sequencing method for unit-load automated storage and retrieval systems. Comput Ind Eng 2012; 63: 980-984.

[16] Ghomri L, Sari Z. Mathematical modeling of the average retrieval time for flow-rack automated storage and retrieval systems. J Manuf Syst 2017; 44: 165-178.

[17] Yang P, Miao L, Xue Z, et al. Variable neighborhood search heuristic for storage location assignment and storage/retrieval scheduling under shared storage in multi-shuttle automated storage/retrieval systems. Transp Res Part E Logist Transp Rev 2015; 79: 164-177.

[18] Brezovnik S, Gotlih J, Balič J, et al. Optimization of an automated storage and retrieval systems by swarm intelligence. In: 25th DAAAM International Symposium on Intelligent Manufacturing and Automation, DAAAM 2014. Elsevier B.V., 2015, pp. 1309-1318.

\section{Anexo 1}

!Modelo de asignación para reducir la distancia total recorrida en las operaciones de ciclo dual

(Almacenamiento-Recolección);

Model:

Sets:

estiva;

recoleccion;

matriz(estiva, recoleccion): distancia,T1,T2,T3;

endsets

data:

!Número de ciclos duales en cada turno;

Tur1 $=11$

Tur2 $=9$

Tur3= 5;

!miembros de los conjuntos; estiva = s1 s2 s3 s4 s5 s6 s7 s8 s9 s10 s11 s12 s13 s14 s15 s16 s17 s18 s19 s20 s21 s22 s23 24 s25; recoleccion $=r 1$ r2 r3 r4 r5 r6 r7 r8 r9 r10 r11 r12 r13 r14 r15 r16 r17 r18 r19 r20 r21 r22 r23 r24 r25;

!Matriz de distancias;

distancia $=$

$\begin{array}{lllllll}27 & 35 & 31 & 40 & 26 & 30 & 36 \\ & 40 & 28 & 31 & 42 & 37 & 40 \\ & 44 & 46 & 44 & 48 & 37 & 39 \\ & 52 & 41 & 49 & 43 & 46 & 47 \\ 41 & 43 & 39 & 48 & \ldots & & \end{array}$

enddata

$\min =@ \operatorname{sum}\left(\operatorname{matriz}(\mathrm{i}, \mathrm{j}): \operatorname{distancia}(\mathrm{i}, \mathrm{j}){ }^{\star} \mathrm{T} 1(\mathrm{i}, \mathrm{j})+\right.$ distancia $\left.(\mathrm{i}, \mathrm{j})^{*} \mathrm{~T} 2(\mathrm{i}, \mathrm{j})+\operatorname{distancia}(\mathrm{i}, \mathrm{j})^{*} \mathrm{~T} 3(\mathrm{i}, \mathrm{j})\right)$;

!Para cada estiva seleccionar sólo un recolección y viceversa;

@for(estiva(i): @sum(recoleccion(j): T1(i,j))<=1);

@for(recoleccion(j): @sum(estiva(i): T1(i,j))<=1);

$@ \operatorname{sum}(\operatorname{matriz}(\mathrm{i}, \mathrm{j}): \mathrm{T} 1(\mathrm{i}, \mathrm{j}))=\operatorname{Tur} 1$;

@for(matriz (i,j): @bin(T1));

@for(estiva(i): @sum(recoleccion(j): T2(i,j))<=1);

@for(recoleccion(j): @sum(estiva(i): T2(i,j))<=1);

@ $\operatorname{sum}(\operatorname{matriz}(\mathrm{i}, \mathrm{j}):$ T2(i,j))=Tur2

@for(matriz (i,j): @bin(T2));

@for(estiva(i): @sum(recoleccion(j): T3(i,j))<=1);

@for(recoleccion(j): @sum(estiva(i): T3(i,j))<=1);

$@ \operatorname{sum}($ matriz $(i, j):$ T3(i,j))=Tur3;

@for(matriz (i,j): @bin(T3));

end 